\title{
Cardiovascular MRI evidence of reduced systolic function and reduced LV mass in rheumatoid arthritis: impact of disease phenotype
}

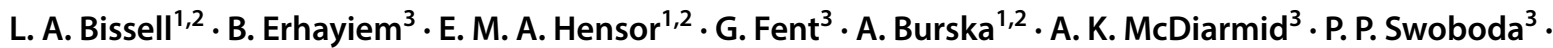 \\ H. Donica ${ }^{4}$. S. Plein ${ }^{3} \cdot$ M. H. Buch ${ }^{1,2} \cdot$ J. P. Greenwood ${ }^{3} \cdot$ J. Andrews ${ }^{1,2}$
}

Received: 11 March 2019 / Accepted: 12 October 2019 / Published online: 8 February 2020

(c) The Author(s) 2020

\begin{abstract}
The accelerated risk of cardiovascular disease (CVD) in Rheumatoid Arthritis (RA) requires further study of the underlying pathophysiology and determination of the at-risk RA phenotype. Our objectives were to describe the cardiac structure and function and arterial stiffness, and association with disease phenotype in patients with established) RA, in comparison to healthy controls, as measured by cardiovascular magnetic resonance imaging (CMR). 76 patients with established RA and no history of $\mathrm{CVD}$ /diabetes mellitus were assessed for RA and cardiovascular profile and underwent a non-contrast 3T-CMR, and compared to 26 healthy controls. A univariable analysis and multivariable linear regression model determined associations between baseline variables and CMR-measures. Ten-year cardiovascular risk scores were increased in RA compared with controls. Adjusting for age, sex and traditional cardiovascular risk factors, patients with RA had reduced left ventricular ejection fraction (mean difference $-2.86 \%(-5.17,-0.55) \mathrm{p}=0.016)$, reduced absolute values of mid systolic strain rate $(\mathrm{p}<0.001)$ and lower late/active diastolic strain rate $(\mathrm{p}<0.001)$ compared to controls. There was evidence of reduced LV mass index $(\mathrm{LVMI})\left(-4.56 \mathrm{~g} / \mathrm{m}^{2}(-8.92,-0.20), \mathrm{p}=0.041\right)$. CMR-measures predominantly associated with traditional cardiovascular risk factors; male sex and systolic blood pressure independently with increasing LVMI. Patients with established RA and no history of CVD have evidence of reduced LV systolic function and LVMI after adjustment for traditional cardiovascular risk factors; the latter suggesting cardiac pathology other than atherosclerosis in RA. Traditional cardiovascular risk factors, rather than RA disease phenotype, appear to be key determinants of subclinical CVD in RA potentially warranting more effective cardiovascular risk reduction programs.
\end{abstract}

Keywords Rheumatoid arthritis · Cardiovascular disease $\cdot$ Cardiovascular MRI

J. Andrews

j.andrews@leeds.ac.uk

L. A. Bissell

1.a.bissell@leeds.ac.uk

B. Erhayiem

B.Erhayiem@leeds.ac.uk

E. M. A. Hensor

e.m.a.hensor@leeds.ac.uk

G. Fent

G.J.Fent@leeds.ac.uk

\section{A. Burska}

A.N.Burska@leeds.ac.uk

A. K. McDiarmid

A.K.McDiarmid@leeds.ac.uk

P. P. Swoboda

P.Swoboda@leeds.ac.uk

H. Donica

donicahelena@gmail.com
S. Plein

S.Plein@leeds.ac.uk

M. H. Buch

m.buch@leeds.ac.uk

J. P. Greenwood

j.greenwood@leeds.ac.uk

1 Leeds Institute of Rheumatic and Musculoskeletal Medicine, University of Leeds, Leeds, UK

2 NIHR Leeds Biomedical Research Centre, Leeds Teaching Hospitals NHS Trust, Leeds, UK

3 Multidisciplinary Cardiovascular Research Centre \& The Division of Biomedical Imaging, Leeds Institute of Cardiovascular and Metabolic Medicine, University of Leeds, Leeds, UK

4 Medical University of Lublin, Lublin, Poland 


\section{Introduction}

Rheumatoid arthritis (RA) is associated with an accelerated risk of cardiovascular disease (CVD), with both traditional cardiovascular risk factors and systemic inflammation playing a role [1]. To improve on the European league against rheumatism (EULAR) recommendations for reducing CVD in RA [2], there is a need for greater understanding of the underlying pathophysiology and determination of the RA phenotype most at risk of CVD.

Cardiovascular magnetic resonance (CMR) imaging provides highly reproducible quantitative assessment of the heart and cardiovascular system, with high diagnostic accuracy for ischaemic heart disease (IHD) [3]. CMR is increasingly used as a research tool in patients with RA, detecting the presence of subclinical disease in populations free of clinical CVD [4-8]. The largest CMR study in RA ( $\mathrm{n}=75$, some with diabetes) reported a $4.4 \%$ reduction in LV ejection fraction (LVEF), but interestingly also a $15 \%$ reduction in LV mass index (LVMI) in those with RA [9]. A preliminary CMR report of 66 patients with treatment-naive early RA also demonstrated a reduced LVMI [10]. Both of these CMR studies stand in contrast to echocardiographic data [11] and smaller CMR studies in RA $[8,12]$ reporting no difference or increase in LV mass. This is of pathophysiological interest given the occurrence of heart failure in RA is not fully attributable to traditional cardiovascular risk factors or the presence of IHD [13].

This exploratory study describes cardiac structure and function and arterial stiffness in patients with established RA free of known CVD and diabetes mellitus, in comparison to healthy controls, as measured by CMR. The study also describes the association of any cardiovascular abnormalities with RA disease phenotype, to provide insight into the patients most at risk of CVD.

\section{Methods}

Consecutive patients with RA attending rheumatology clinics between January 2011 and September 2014 at the Leeds Teaching Hospitals NHS Trust were considered for this study. Patients were eligible if between 18 and 80 years old, met 1987 ACR criteria [14], had disease for 5 years or more and no history of CVD or diabetes mellitus. Healthy controls, with no history of RA or osteoarthritis affecting their mobility, were mainly identified by asking patients with RA to 'bring a friend'. This study had full ethical approval; REC 09/H1307/98 and REC 10/ H1307/103, NRES Leeds West ethics committee. Following written informed consent obtained according to the
Declaration of Helsinki, study participants were invited to undergo a cardiovascular clinical assessment, fasting blood collection and CMR.

\section{Clinical assessment}

A clinical evaluation recorded demographic data, traditional cardiovascular risk factors and for patients with RA, disease phenotype including 3-variable DAS28-C-reactive protein (CRP) [15]) and Health Assessment QuestionnaireDisease Index [16]. Fasting lipid profile and glucose were measured, and rheumatoid factor (RF), anti-cyclic citrullinated peptide antibody (ACPA), CRP, erythrocyte sedimentation rate in those with RA. Additional blood samples were processed and stored (some at $-30{ }^{\circ} \mathrm{C}$ and some at $-80^{\circ} \mathrm{C}$ ) for later analysis at the Department of Biochemical Diagnostics, Medical University of Lublin, Poland; glucose (using biochemical analyser Cobas INTEGRA 400); N-terminal pro-brain natriuretic peptide (NT-proBNP) (using Cobas 6000 (immunochemistry module Cobas e601)) and insulin (using COBAS e 411 (Roche Diagnostics GmbH, Mannheim, Germany) and appropriate Roche Diagnostics assays). Homeostasis model assessment-estimated insulin resistance (HOMA-IR) was then calculated (fasting insulin $(\mu \mathrm{U} / \mathrm{ml}) \times$ fasting glucose $(\mathrm{mg} / \mathrm{dl}) / 405)[17]$.

\section{CMR imaging}

The CMR study was performed on a 3T Philips Achieva system TX equipped with a 32-channel coil. Low resolution survey, reference scans and localisers determined the cardiac short axis, vertical long axis and horizontal long axis with cine imaging (balanced steady state free precession (SSFP) acquisition, see Fig. 1). LV dimensions and function were obtained from cines covering the entire heart in the LV short axis [18, 19] (balanced SSFP, multiphase, contiguous slices, voxel size $1.2 \times 1.2 \times 10 \mathrm{~mm}^{3}, 50$ cardiac phases).

For aortic distensibility, high temporal resolution sagitaloblique and transverse cines were acquired to measure the diameter and area of the ascending aorta, descending aorta and aortic arch at the level of the main pulmonary artery [20]. Blood pressure was recorded immediately prior to image acquisition. Aortic pulse wave velocity (PWV) was assessed using identical geometry planning with retrospectively gated, through-plane, phase-contrast velocity encoded images (breath-hold, single slice, $10 \mathrm{~mm}$ thick, 50 phases, VENC $200 \mathrm{~cm} / \mathrm{s}$ ).

Tissue tagging for strain analysis and diastology were generated from the basal, mid and apical LV using the 3-of$5^{\prime}$ approach [21] (spatial modulation of magnetization pulse sequence, spatial resolution $1.51 \times 1.57 \times 10 \mathrm{~mm} 3$, tag separation $7 \mathrm{~mm}, \geq 18$ phases, typical TR/TE $5.8 / 3.5 \mathrm{~ms}$, flip angle $10^{\circ}$ ). 
Fig. 1 Typical CMR images from this study including SSFP cine imaging planned in the four chamber view (upper left), high temporal resolution cine imaging for aortic distensibility (lower left), native T1 mapping at mid ventricular level (upper right) and tagged cine imaging for strain analysis (lower right)
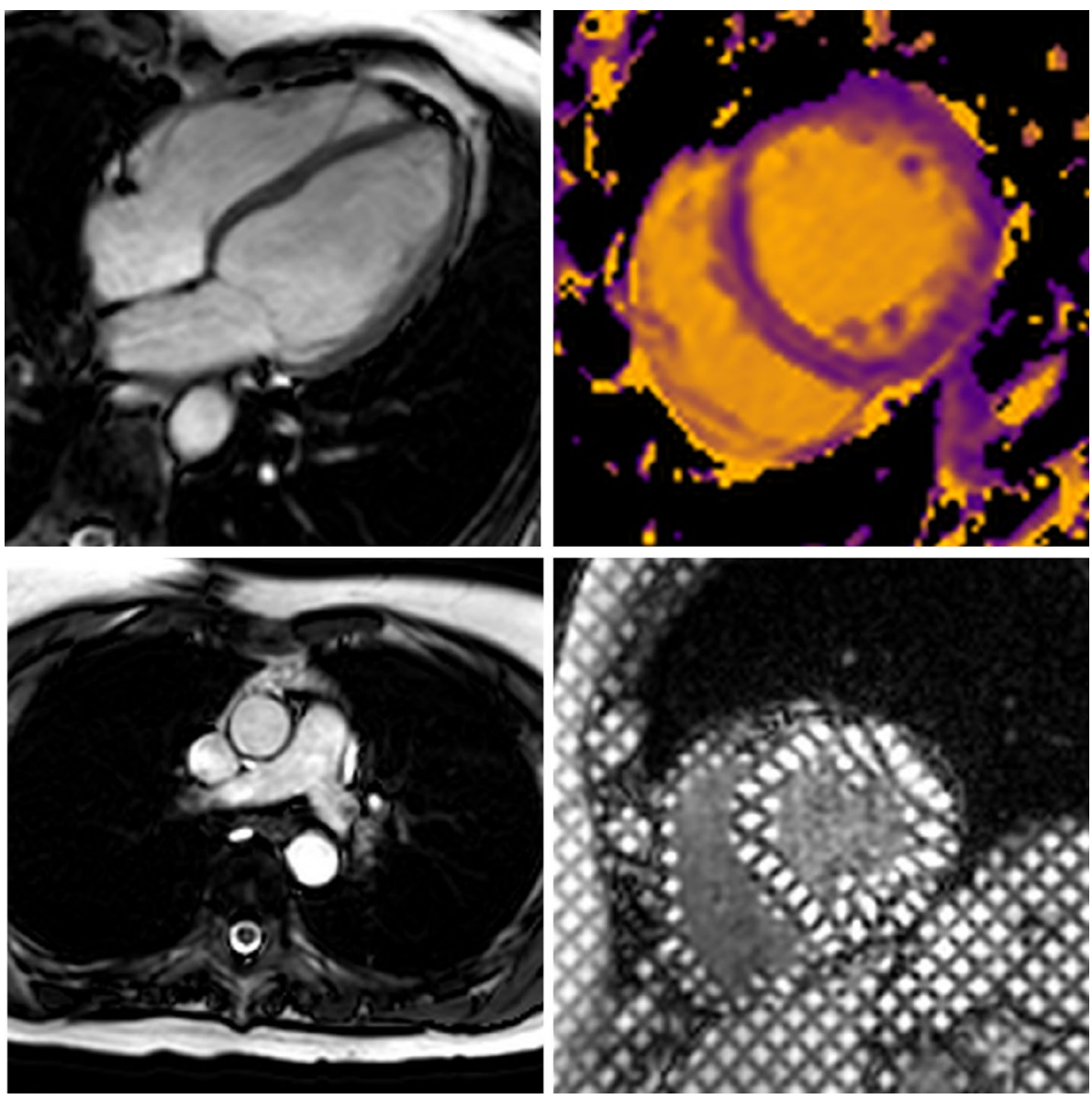

Native T1 mapping was acquired with a single breathhold mid-slice in the LV short axis using an electrocardiogram-triggered modified Look-Locker inversion method to acquire 11 images (3-3-5 acquisition with $3 \times \mathrm{R}-\mathrm{R}$ interval recovery epochs, voxel size $1.7 \times 2.14 \times 10 \mathrm{~mm}^{3}$ Trigger delay at end-diastole, flip angle 35o, FOV 320-420 mm) $[22,23]$.

\section{Data analysis}

Image analysis was performed off-line blinded to patient characteristics using CMR42 (CVI42 v4.1.3, Circle Cardiovascular Imaging Inc., Calgary, Canada) and in accordance to recognised reporting standards [24]. LV dimensions and function (excluding papillary muscles) were calculated using standard criteria to delineate cardiac borders [24].

Aortic cross-sectional measurements were made by manual planimetry of the endovascular-blood pool interface, at maximal and minimal distension of the aorta. Aortic distensibility $($ strain/(pulse pressure $(\mathrm{mmHg})) \times 1000)$ [25], and aortic PWV (distance between area of ascending aorta and area of descending aorta/transit time for wave to cover distance) $[25,26]$ were calculated. Analysis was performed using in-house software (PMI 0.4) based on IDL 6.4 (ITT Visual Information Systems, Boulder, CO, USA) [27].

Strain analysis and LV torsion from the tagging series was derived using the open source software Osirix inTag (http://www.osrix-viewer.com). LV twist was calculated by subtracting the basal from apical rotation [28]. LV torsion takes the heart radius and length into account, describing it as the circumferential-longitudinal shear angle making the measurement comparable between hearts of different sizes and is related to myocardial fibre orientation [29]; calculated by peak twist $\times($ apical radius + basal radius $)) /(2 \times$ apex to base length).

Native myocardial T1 was measured from a desired region of interest (ROI) in the mid-ventricular wall [30]. The ROI was manually motion corrected as required from source images and care taken to avoid partial-volume effects from neighbouring tissue or blood pool.

\section{Statistical analysis}

The statistical packages SPSS (IBM SPSS Statistics 22) and Stata/IC 13.1 were used. Following a descriptive analysis, independent t-tests determined unadjusted differences between patients with RA and controls. Linear regression 
determined differences when adjusted for age, sex and cardiovascular risk factors [defined as hypertension (either history of or on anti-hypertensive agent), dyslipidaemia (either history of or on lipid-lowering medication or total cholesterol/high-density lipoprotein cholesterol (TC/HDL-C) ratio greater than 6) and ever smoked]. Non-normally distributed variables (HOMA-IR and NT-proBNP) were log-transformed prior to analysis. Ordinal JBS2 scores were compared using Mann-Whitney U test. Using Holm's method to correct for multiple comparisons [31], the threshold for statistical significance at the $5 \%$ level was set to $\mathrm{p}<0.016$.

Within the RA group, Pearson's/Spearman's correlation/ univariable analyses were used to determine associations between baseline variables and CMR-measures, using logtransformed values when appropriate. Any variables considered to be associated with CMR-measures in the literature or strongly correlating by univariate analysis (coefficient greater than 0.3 ) were entered into a multivariable linear regression model.

In the event of missing serology the most recent value preceding the visit was carried forward into the data, excluding CRP due to its capacity to vary or for lipid/glucose profile, as a fasting state could not be verified.

\section{Results}

Ninety-five consecutive patents with RA were recruited; 76 of these underwent CMR. Thirty healthy controls were recruited and 26 of these underwent CMR. Reasons for not having CMR performed included claustrophobia $(\mathrm{RA} n=6)$, difficulty in contacting patient for CMR (RA $n=7$, controls $n=3$ ) or patient changing their mind (RA $n=3$, controls $\mathrm{n}=1$ ), non-MR compatible implant ( RA $n=1$ ), patient too large for scanner $(\mathrm{RA} n=1)$ and incomplete study data retrieved $(n=1)$.

\section{Study participant characteristics}

Of those who underwent a CMR scan, Tables 1 and 2 outline the demographic, cardiovascular risk profile and RA diseasespecific features. The mean age (standard deviation, SD) of patients with RA was 60 (9.2) years, 74\% were female and $95 \%$ white. Median (interquartile range, IQR) disease duration was $16.5(10.7,25.7)$ years, $90 \%$ were seropositive for RF or ACPA and $78 \%$ had erosive disease. Patients overall were in remission; median (IQR) 3variable-DAS28 2.39 (1.15, 3.36). A significant proportion had cardiovascular risk factors, including 33\% with hypertension. The control group were younger (mean (SD) age 51.8 (11.8) years) with fewer $(54 \%)$ females and fewer cardiovascular risk factors.
There was little difference in lipid, glucose levels, HOMA-IR and NT-proBNP levels between the groups (see Table 1). Joint British Societies-2 (JBS2) 10-year cardiovascular risk scores in patients with RA were double that of the controls (not statistically significant); median (IQR) $(8.6(4.1,8.6) \%$ versus $4.2(1.2,10.5) \%$ in controls $(\mathrm{p}=0.087)$; a significant difference seen when following EULAR guidelines (multiply risk scores by 1.5 in patients with RA [2]); median JBS2 risk difference 5.7 (95\% CI $2.7,10.2) \mathrm{p}=0.003$ in RA.

In patients with $\mathrm{RA}$, TC/HDL-C ratio was not associated with C-reactive protein, 3-variable DAS28, ACPA or RA disease duration (data not shown); with similar findings excluding patients on a statin $(n=12)$. There were weak, positive associations between NT-proBNP and both age and RA disease duration $(r=0.325, p=0.006$, and $\mathrm{r}=0.278, \mathrm{p}=0.019$ respectively). There were also weak, positive associations between HOMA-IR and both body mass index and waist/hip circumference ratio $(r=0.240$, $\mathrm{p}=0.044$ and $\mathrm{r}=0.368, \mathrm{p}=0.002$ respectively).

\section{Cardiovascular magnetic resonance imaging}

\section{Differences between patients and controls}

The CMR results are shown in Table 3. No pericardial effusions were noted. No significant valvular pathology or cardiac masses or features of cardiomyopathy were detected, although flow imaging and post-contrast imaging were not performed. Patients with RA demonstrated a reduction in absolute values for mid systolic strain rate (mid S') reducing further in the analysis adjusted for age, sex and cardiovascular risk factors $(0.227(0.104,0.349)$, $\mathrm{p}<0.001)$. They also demonstrated a reduction in LVEF (mean (SD) in RA 59.1 (4.6) vs. 59.7 (4.8) \% in controls); borderline significant in the adjusted analysis (mean difference $-2.858(-5.167,-0.550) \%, p=0.016)$. Although early diastolic strain rate was similar across the groups, active/late diastolic strain rate was reduced in those with $\mathrm{RA}$ in the adjusted analysis $(-0.45(-0.67,-0.23)$, $\mathrm{p}<0.001)$.

LVMI was reduced in patients with RA (mean (SD) $36.35(10.52)$ vs. $44.06(14.49) \mathrm{g} / \mathrm{m}^{2}$ in controls, $\left.\mathrm{p}=0.005\right)$, and when adjusted for age and sex, but not after further adjustment for cardiovascular risk factors, although the difference remained substantive (mean difference $(95 \% \mathrm{CI})$ $-4.56(-8.92,-0.199) \mathrm{p}=0.041)$.

Whilst aortic distensibility was lower in RA in the unadjusted analysis, there was no substantive/statistical difference in the adjusted analysis between the groups. The remaining differences were substantive but did not meet the revised threshold for statistical significance. 
Table 1 Study participant characteristics

\begin{tabular}{|c|c|c|c|}
\hline Variable & Expressed as & $\begin{array}{l}\text { RA patients } \\
\mathrm{n}=76\end{array}$ & $\begin{array}{l}\text { Controls } \\
\mathrm{n}=26\end{array}$ \\
\hline \multicolumn{4}{|l|}{ Demographics } \\
\hline Age (years) & Mean, SD & $60(9.2)$ (range $31-78)$ & $52.2(11.4)$ (range $35-80)$ \\
\hline Female & n $\%$ & $56(73.7)$ & $14(53.8)$ \\
\hline White & $\mathrm{n} \%$ & $72(94.7)$ & $23 / 24(95.8)$ \\
\hline \multicolumn{4}{|l|}{$\mathrm{CV}$ risk profile } \\
\hline PMH hypertension & n $\%$ & $25(32.9)$ & 2/23 (8.7) \\
\hline PMH Hypercholesterolaemia & n $\%$ & $19(25)$ & $1 / 23(4.3)$ \\
\hline \multicolumn{4}{|l|}{ Smoking status } \\
\hline Never & n $\%$ & $35(46.1)$ & $12 / 23(52.2)$ \\
\hline Ex & & $31(40.8)$ & $9 / 23(39.1)$ \\
\hline Current & & $10(13.2)$ & $2 / 23(8.7)$ \\
\hline Alcohol intake (units/week) & Median (IQR) & $2(2,8)$ & $2(2,8)$ \\
\hline FHx premature CVD* & $\mathrm{n} \%$ & $20(26.3)$ & $4 / 22(18.2)$ \\
\hline $\begin{array}{l}\text { Five or more fruit/vegetables daily intake (days/ } \\
\text { week) }\end{array}$ & Median (IQR) & $5(4,7)$ & $5(4,7)$ \\
\hline Moderate exercise (mins/week) & Median (IQR) & $37.5(0,142.5)$ & $60(0,255)$ \\
\hline Number of current anti-hypertensives & $\mathrm{n} \%$ & $\begin{array}{l}10(13.2) \text { on } 1 \text { drug } \\
8(10.5) \text { on } 2 \text { drugs } \\
2(2.6) \text { on } 3 \text { drugs }\end{array}$ & $1 / 23(4.3)$ on 2 drugs \\
\hline Current use of statin & n $\%$ & $12(15.8)$ & $1 / 23(4.3)$ \\
\hline BMI & Mean, SD & $26.1(3.5)$ & $25.0(3.4)$ \\
\hline Waist/hip ratio & Mean, SD & $0.84(0.08)(\mathrm{n}=74)$ & $0.82(0.09)(\mathrm{n}=23)$ \\
\hline Systolic BP (mmHg) & Mean, SD & $135(20)$ & $127(16)(\mathrm{n}=25)$ \\
\hline Diastolic BP (mmHg) & Mean, SD & $80(12)$ & $72(10)(n=25)$ \\
\hline \multicolumn{4}{|l|}{ Fasting blood collection } \\
\hline Fasting TC/HDL-C ratio & Mean, SD & $3.4(1.0)(n=73)$ & $3.3(1.0)(\mathrm{n}=21)$ \\
\hline Fasting total cholesterol, $\mathrm{mmol} / \mathrm{L}$ & Mean, SD & $5.3(1.1)(n=75)$ & $5.1(0.9)(\mathrm{n}=21)$ \\
\hline Fasting HDL-C, mmol/L & Mean, SD & $1.7(0.4)(\mathrm{n}=73)$ & $1.6(0.4)(\mathrm{n}=21)$ \\
\hline Fasting LDL-C, mmol/L & Mean, SD & $3.1(0.9)(n=73)$ & $3.0(0.9)(\mathrm{n}=21)$ \\
\hline HOMA-IR & Geometric mean & $1.10(\mathrm{n}=71)$ & $1.20(\mathrm{n}=22)$ \\
\hline NT-proBNP, pg/ml & Geometric mean & $57.64(\mathrm{n}=71)$ & $42.17(n=22)$ \\
\hline
\end{tabular}

$B M I$ body mass index, $B P$ blood pressure, $C V D$ cardiovascular disease, FHx family history of, HOMA-IR homeostasis model of assessment of insulin resistance, $P M H$ past medical history of, $N T$-proBNP N-terminal pro-brain natriuretic peptide, $R A$ rheumatoid arthritis, $T C / H D L-C$ total cholesterol/high-density lipoprotein cholesterol ratio

*First degree relative with history of CVD when 60 years old or younger if relative female, and 55 years old or younger if relative male

\section{Association with RA disease phenotype and soluble cardiovascular biomarkers}

LVEF and LVMI were analysed further given the differences seen between the groups and provision of information on structure/function. A univariate analysis (Table 4) in those with RA found that male sex, systolic blood pressure (sysBP) and waist/hip circumference ratio were associated with increasing LVMI, with male sex and sysBP independently associated with LVMI in a multivariable linear regression analysis (MVA). Although no variables were associated with LVEF on univariate analysis (Table 5), male sex was associated with LVEF in a MVA. No RA-specific features were associated with CMR-outcomes.

\section{Discussion}

In a population of established RA free of CVD and diabetes mellitus, combining clinical assessment with measurements of soluble biomarkers of CVD and CMR, this study reports a reduction in systolic function (LVEF, peak mid systolic strain rate), early/mid diastolic strain rate and LVMI after controlling for age, sex and traditional cardiovascular risk factors, and the association of surrogate measures of CVD 
Table 2 Disease specific characteristics of patients with rheumatoid arthritis

\begin{tabular}{|c|c|c|}
\hline RA phenotype & Data as expressed & $\begin{array}{l}\text { RA patients } \\
\mathrm{n}=76\end{array}$ \\
\hline Disease duration (years) & Median (IQR) & $16.5(10.7,25.7)($ range $4.2,43.4)$ \\
\hline Early morning stiffness (mins) & Median (IQR) & $10(10,37.5)$ \\
\hline History of orthopaedic joint surgery & n $\%$ & $21(27.6)$ \\
\hline Number of orthopaedic joint surgical episodes & n $\%$ & $\begin{array}{l}10(13.2)-1 \text { episode } \\
3(3.9)-2 \text { episodes } \\
6(7.9)-3 \text { episodes } \\
2(2.6)-4 \text { episodes }\end{array}$ \\
\hline Current use of oral prednisolone & $\mathrm{n} \%$ & $4(5.3)$ \\
\hline Current use of non-biological DMARD & $\mathrm{n} \%$ & $62(81.6)$ \\
\hline Number of csDMARDs currently taking & n \% & $\begin{array}{l}48(63.2) \text { taking } 1 \\
8(10.5) \text { taking } 2 \\
8(10.5) \text { taking } 3\end{array}$ \\
\hline Number of previously tried csDMARDs & Median (IQR) & $2(1,3)($ range 0,7$)$ \\
\hline Current use of biological DMARD & $\mathrm{n} \%$ & $51(67.1)$ \\
\hline Current TNFI users & & $22(27.6)$ \\
\hline Current Rituximab users & & $26(34.2)$ \\
\hline Current Tocilizumab users & & $3(3.9)$ \\
\hline Current Abatacept users & & $1(1.3)$ \\
\hline Number of treatment cycles in current RTX users & Median (IQR) & $4(3,5.25)($ range 1,9$)$ \\
\hline Number of previously tried biological DMARDs & Median (IQR) & $0(0,1)$ \\
\hline Patient general health VAS & Median (IQR) & $31(15,52)$ \\
\hline 28-Tender joint count & Median (IQR) & $2(0,6)$ \\
\hline 28-Swollen joint count & Median (IQR) & $0(0,1)$ \\
\hline HAQ-DI & Median (IQR) & $1.44(0.53,2.00)$ \\
\hline 3 variable DAS28CRP & Median (IQR) & $2.39(1.15,3.36)$ \\
\hline Erosions on hands/feet radiograph & $\mathrm{n} \%$ & $57 / 73(78.1)$ \\
\hline $\mathrm{CRP}(\mathrm{mg} / \mathrm{L})$ & Median (IQR) & $<5(0,7.8)$ \\
\hline $\operatorname{ESR}(\mathrm{mm} / \mathrm{h})$ & Median (IQR) & $14(6,27)$ \\
\hline Rheumatoid factor positive $(\geq 40 \mathrm{iu} / \mathrm{ml})$ & $\mathrm{n} \%$ & $53(69.7)$ \\
\hline ACPA positive $(\geq 10 \mathrm{U} / \mathrm{ml})$ & n $\%$ & $61 / 75(81.3)$ \\
\hline
\end{tabular}

$A C P A$ anti-citrullinated peptide antibody, CRP C-reactive protein, $c s D M A R D s$ conventional synthetic DMARDs, DAS28CRP 28-joint disease activity score, DMARDs disease-modifying anti-rheumatic drugs, $E S R$ erythrocyte sedimentation rate, $H A Q-D I$ health assessment questionnaire-disability index, $R T X$ rituximab, VAS visual assessment score with traditional cardiovascular risk factors rather than RA disease-specific features.

The only similar sized CMR study in RA $(n=75)$ by Giles et al. also reported a $4.4 \%$ reduction in LVEF and $15 \%$ reduction in LVMI in the adjusted mean values in patients free of known CVD compared to controls $(n=225)$, after adjustment for blood pressure, heart rate, HDL-C, triglycerides, habitual exercise and coronary calcium score [9]. Although age, smoking habits and statin use in their study were comparable to our study, there were fewer females $(52 \%)$, fewer white $(88 \%)$, greater burden of hypertension (55\%) and importantly, inclusion of patients with diabetes (4\%). Their patients with RA may have been subjected to a smaller burden of inflammation than in our study (shorter disease duration and fewer on biological disease modifying anti-rheumatic drugs (49\%)) however they were not in remission (mean DAS28 3.51). Our study demonstrates similar results in patients free of diabetes with a reduction in the adjusted mean values in LVEF of $5.11 \%$ (intercept $55.884, \mathrm{~B}-2.858$ (95\% CI $-5.167,-0.550) \mathrm{p}=0.016$ ) and LVMI of $15.5 \%$ (intercept $29.456, \mathrm{~B}-4.558(95 \% \mathrm{CI}-8.917,-0.199) \mathrm{p}=0.041)$ for patients with RA, who were overall in remission, i.e. CMR abnormalities despite no active RA disease.

Other CMR studies to date reporting LVEF have been inconsistent involving smaller cohorts [12] and shorter disease duration [8]. Although Ntusi et al. reported similar LVEF, they did determine reduced peak systolic circumferential strain and reduced diastolic strain rate in those with RA [8]. Similarly, smaller CMR studies have not described reduced LV mass [8, 12], although our research 
Table 3 Cardiovascular magnetic resonance imaging measures in study participants

\begin{tabular}{|c|c|c|c|c|c|}
\hline Variable & $\begin{array}{l}\text { RA patients } \\
\mathrm{n}=76\end{array}$ & $\begin{array}{l}\text { Controls } \\
\mathrm{n}=26\end{array}$ & $\begin{array}{l}\text { Unadjusted } p \\
\text { value for differ- } \\
\text { ence }\end{array}$ & $\begin{array}{l}\text { Mean difference }(95 \% \\
\mathrm{CI}), \mathrm{p} \text { value adjusted } \\
\text { for age and sex }\end{array}$ & $\begin{array}{l}\text { Mean difference (95\%), } \\
\text { p value adjusted for age/ } \\
\text { sex/cardiovascular risk } \\
\text { factors* }\end{array}$ \\
\hline $\begin{array}{l}\text { LV ejection fraction } \\
(\%)\end{array}$ & $59.1(4.6)(\mathrm{n}=74)$ & $59.7(4.8)$ & 0.560 & $\begin{array}{l}-2.100(-4.268 \\
0.068), 0.057\end{array}$ & $\begin{array}{l}-2.858(-5.167 \\
-0.550) 0.016\end{array}$ \\
\hline LV EDV index $\left(\mathrm{ml} / \mathrm{m}^{2}\right)$ & $79.09(14.59)(\mathrm{n}=74)$ & $91.08(24.32)$ & 0.024 & $\begin{array}{l}-5.15(-12.908,1.878) \\
0.142\end{array}$ & $\begin{array}{l}-4.683(-12.275 \\
2.909) 0.224\end{array}$ \\
\hline LV ESV index $\left(\mathrm{ml} / \mathrm{m}^{2}\right)$ & $32.45(7.60)(\mathrm{n}=74)$ & $36.88(12.00)$ & 0.032 & $\begin{array}{l}-0.545(-4.311,3.222) \\
0.775\end{array}$ & $\begin{array}{l}1.187(-2.491,4.865) \\
0.523\end{array}$ \\
\hline LV mass index $\left(\mathrm{g} / \mathrm{m}^{2}\right)$ & $36.35(10.52)(\mathrm{n}=74)$ & $44.06(14.49)$ & 0.005 & $\begin{array}{l}-5.421(-9.615 \\
-1.227), 0.012\end{array}$ & $\begin{array}{l}-4.558(-8.917 \\
-0.199) 0.041\end{array}$ \\
\hline LV mass/EDV (g/ml) & $0.46(0.10)(\mathrm{n}=74)$ & $0.48(0.10)$ & 0.266 & $\begin{array}{l}-0.035(-0.075, \\
0.005), 0.086\end{array}$ & $\begin{array}{l}-0.033(-0.075,0.010) \\
0.129\end{array}$ \\
\hline $\begin{array}{l}\text { Stroke volume index } \\
\left(\mathrm{ml} / \mathrm{m}^{2}\right)\end{array}$ & $46.29(7.26)(\mathrm{n}=74)$ & $53.47(11.20)$ & 0.005 & $\begin{array}{l}-4.273(-7.998 \\
-0.548), 0.025\end{array}$ & $\begin{array}{l}-3.551(-7.516,0.413) \\
0.078\end{array}$ \\
\hline Peak mid systolic strain & $\begin{array}{l}-0.225(0.037) \\
(\mathrm{n}=69)\end{array}$ & $\begin{array}{l}-0.244(0.033) \\
\quad(n=23)\end{array}$ & 0.032 & $\begin{array}{l}0.020(0.002,0.039) \\
0.032\end{array}$ & $\begin{array}{l}0.019(-0.001,0.040) \\
0.066\end{array}$ \\
\hline Mid S’ & $\begin{array}{l}-1.205(0.209) \\
\quad(\mathrm{n}=69)\end{array}$ & $\begin{array}{l}-1.398(0.237) \\
(\mathrm{n}=23)\end{array}$ & $<0.001$ & $\begin{array}{l}0.200(0.089,0.311) \\
0.001\end{array}$ & $\begin{aligned} 0.227 & (0.104 \\
0.349) & <0.001\end{aligned}$ \\
\hline Mid E' & $0.70(0.21)(n=69)$ & $0.69(0.29)(n=23)$ & 0.794 & $\begin{array}{l}0.02(-0.003,0.01) \\
0.791\end{array}$ & $\begin{array}{l}0.04(-0.09,0.17) \\
0.511\end{array}$ \\
\hline Mid A' & $1.65(0.36)(\mathrm{n}=68)$ & $2.11(0.50)(\mathrm{n}=23)$ & $<0.001$ & $\begin{array}{r}-0.44(-0.64, \\
-0.45),<0.001\end{array}$ & $\begin{array}{r}-0.45(-0.67 \\
-0.23),<0.001\end{array}$ \\
\hline Peak twist (degrees) & $10.77(4.31)(\mathrm{n}=69)$ & $12.38(3.74)(n=23)$ & 0.113 & $\begin{array}{l}-2.026(-4.162,0.110) \\
0.063\end{array}$ & $\begin{array}{l}-2.451(-4.823 \\
-0.080) 0.043\end{array}$ \\
\hline Torsion (degrees) & $10.94(3.86)(n=68)$ & $10.80(4.41)(n=23)$ & 0.886 & $\begin{array}{l}-0.284(-2.331,1.764) \\
0.784\end{array}$ & $\begin{array}{l}-0.889(-3.139,1.360) \\
0.434\end{array}$ \\
\hline $\begin{array}{l}\text { Native (inferoseptal) } \\
\mathrm{T} 1=(\mathrm{ms})\end{array}$ & $1156.82(53.07)$ & $1186.00(49.30)$ & 0.017 & $\begin{array}{l}-30.09(-56.30 \\
-3.88) 0.025\end{array}$ & $\begin{array}{l}-34.78(-64.09 \\
-5.50) 0.021\end{array}$ \\
\hline $\begin{array}{l}\text { Pulse wave velocity } \\
(\mathrm{m} / \mathrm{sec})\end{array}$ & $7.8(2.9)(\mathrm{n}=73)$ & $7.2(2.3)$ & 0.339 & $\begin{array}{l}-0.289(-1.483,0.905) \\
0.632\end{array}$ & $\begin{array}{l}-0.443(-1.639,0.753) \\
0.464\end{array}$ \\
\hline $\begin{array}{l}\text { Aortic distensibility } \\
\left(10^{-3} \mathrm{mmHg}^{-1}\right)\end{array}$ & $2.60(1.82)(\mathrm{n}=75)$ & $3.83(1.56)(n=25)$ & 0.003 & $\begin{array}{l}-0.340(-0.996,0.316) \\
0.307\end{array}$ & $\begin{array}{l}-0.222(-0.936,0.492) \\
0.538\end{array}$ \\
\hline
\end{tabular}

Bold values represent statistical significance

Values expressed as mean (SD) unless stated otherwise

$A^{\prime}$ late/active diastolic strain rate, $E$ ' early diastolic strain rate, $E D V$ end-diastolic volume, $E S V$ end-systolic volume, $L V$ left ventricular, $S^{\prime}$ peak systolic strain rate

${ }^{*} \mathrm{CV}$ risk factors: hypertension (history/anti-hypertensive agent), dyslipidaemia (history/lipid-lowering medication/TC/HDL-C ratio $>6$ ), ever smoked, premature CVD family history. Using Holm's method for multiple comparisons correction, threshold for statistical significance at 5\% level set to $\mathrm{p}<0.016$

group has reported preliminary findings of lower LV mass, in treatment-naive early RA patients $(n=66)[10]$.

Echocardiography studies have repeatedly reported increased LV mass in patients with RA [32], and often no difference in LVEF $[11,33]$, although reduced LVEF has been associated with active disease [33]. CMR has now demonstrated greater reproducibility over echocardiography in determination of LV systolic function [34] and greater sensitivity to detect small differences in LVEF and LV mass [35], and is now widely accepted as the reference standard $[34,36]$.
The reason for reduced LVMI in RA is unclear, especially given that LV mass usually increases with cardiovascular risk [36]. Indeed, the ten-year cardiovascular risk scores in this study correlated strongly with increasing LV mass (rho $=0.473$ for JBS2 scores, $\mathrm{p}<0.001$ ). Possible causes for lower LVMI in RA patients could include physical deconditioning, myocarditis [9], microvascular dysfunction [37], or even cardiac remodelling [38] given abnormal geometry in RA has been reported previously [39]. The reduction in LVMI may also reflect the pathological processes of 'RAcachexia' (loss of muscle mass with increase in fat mass) [40]. Although RA cachexia is a cytokine driven process, 
Table 4 Univariable and multivariable analysis of variables associated with CMR measured LVMI

\begin{tabular}{|c|c|c|c|c|c|}
\hline \multirow[t]{3}{*}{ Variable } & \multicolumn{5}{|l|}{ LVMI } \\
\hline & \multicolumn{3}{|c|}{$\begin{array}{l}\text { Univariable analysis } \\
\text { (number of observations }=74 \text {, unless otherwise stated) }\end{array}$} & \multicolumn{2}{|l|}{$\begin{array}{l}\text { Multivariable analysis } \\
\mathrm{R}^{2}=0.601, \mathrm{n}=68\end{array}$} \\
\hline & $\begin{array}{l}\text { Correlation } \\
\text { coefficient }\end{array}$ & B $(95 \%$ CI $)$ & $\mathrm{p}$ value & B $(95 \%$ CI $)$ & $\mathrm{p}$ value \\
\hline Age* & 0.139 & $0.157(-0.106,0.420)$ & 0.238 & $0.006(-0.215,0.228)$ & 0.955 \\
\hline Male sex* & 0.665 & $15.657(11.50,19.783)$ & $<0.001$ & $14.764(-10.00,19.528)$ & $<0.001$ \\
\hline Systolic blood pressure* & 0.344 & $0.184(0.066,0.302)$ & 0.003 & $0.122(0.027,0.217)$ & 0.013 \\
\hline Ever smoked* & 0.186 & $3.905(-0.948,8.758)$ & 0.113 & $3.759(0.232,7.285)$ & 0.037 \\
\hline Body mass index & 0.005 & $0.017(-0.738,0.773)$ & 0.964 & - & - \\
\hline Waist/hip circumference & 0.327 & $43.661(13.571,73.750)(n=72)$ & 0.005 & $3.899(-22.997,30.795)$ & 0.773 \\
\hline TC/HDL-C $*$ & 0.059 & $0.608(-1.874,3.091)(\mathrm{n}=71)$ & 0.626 & $0.278(-1.462,2.018)$ & 0.750 \\
\hline HOMA-IR & 0.137 & $0.642(-0.489,1.773)(\mathrm{n}=69) \dagger$ & 0.261 & - & - \\
\hline NT-proBNP & 0.062 & $0.009(-0.27,0.045)(\mathrm{n}=69)$ & 0.615 & - & - \\
\hline RA disease duration* & 0.172 & $0.175(-0.060,0.411)$ & 0.142 & $0.046(-0.133,0.224)$ & 0.612 \\
\hline 3 Variable DAS28 & 0.042 & $0.341(-1.585,2.268)$ & 0.725 & - & - \\
\hline ACPA* & -0.156 & $-4.2121(10.294,2.053)(n=73)$ & 0.187 & $2.014(-2.559,6.587)$ & 0.382 \\
\hline HAQ-DI & -0.140 & $-1.883(-5.097,1.331)(\mathrm{n}=70)$ & 0.247 & - & - \\
\hline History of joint surgery & 0.112 & $2.587(-2.825,7.998)$ & 0.344 & - & - \\
\hline Current use of biological DMARD & -0.008 & $-0.169(-5.413,5.075)$ & 0.949 & - & - \\
\hline
\end{tabular}

Bold values represent statistical significance

$A C P A$ anti-citrullinated peptide antibody, $C R P$ C-reactive protein, DAS28CRP 28-joint disease activity score, DMARD disease-modifying antirheumatic drug, HAQ-DI health assessment questionnaire-disability index, HOMA-IR homeostasis model of assessment of insulin resistance, LVMI LV mass index, NT-proBNP N-terminal pro-brain natriuretic peptide, $T C / H D L-C$ total cholesterol/high-density lipoprotein cholesterol ratio

*Entered into linear regression model as associated with LV mass in the literature

$\dagger$ Excluding high outlier: Correlation coefficient B $-0.025(95 \% \mathrm{CI}-2.732,2.195) \mathrm{p}=0.828$

studies fail to demonstrate its improvement following suppression of disease activity [40].

This study also demonstrated that traditional cardiovascular risk factors associated with surrogate measures of subclinical CVD, rather than RA specific features. The European Guidelines on CVD prevention provide clear recommendations applicable to those with RA [41], with lower cardiovascular event rates associated with improvement of cardiovascular risk factors in RA [42]; however, in practice, the management of cardiovascular risk in RA is suboptimal [43]. This should therefore become a priority for the reduction of CVD in RA.

\section{Limitations}

Although relatively large compared to previous studies, this exploratory study was not powered, however, we feel the findings are worth further consideration and validation. This cross-sectional study was also unable to quantify the burden of inflammation patients with RA were exposed to over time. The cohort had established severe disease with many requiring multiple/biological disease-modifying antirheumatic drugs, however, they were also in remission. In the absence of previous regular measures of disease activity/ inflammation, disease duration in this cohort is the proxy measure for burden of disease. The cumulative exposure to corticosteroids (known to increase LV mass) was not quantified and it was not possible to measure the effect of DMARDs, although published data suggests DMARDs can reduce arterial stiffness and $\mathrm{CV}$ risk most likely through the reduction of disease activity and systemic inflammation [2].

We were surprised to see a trend for reduced native (inferoseptal) T1 in those with RA. We repeated the analysis for global T1 (average of all myocardial segments) measurements and found no such difference between the groups (1111.80 (52.35) in RA vs. 1111.47 (53.37) in controls, $\mathrm{p}=0.978$. We acknowledge the addition of post-contrast $\mathrm{T} 1$ measurements to derive extra-cellular volume, would have provided greater detail on myocardial tissue composition. Future research agenda.

Larger and longitudinal studies of CMR in RA are required to validate these findings, determine at which point the changes occur, and if they are amenable for improvement 
Table 5 Univariable and multivariable analysis of variables associated with CMR measured LVEF

\begin{tabular}{|c|c|c|c|c|c|}
\hline \multirow[t]{3}{*}{ Variable } & \multicolumn{5}{|l|}{ LVEF } \\
\hline & \multicolumn{3}{|c|}{$\begin{array}{l}\text { Univariate analysis } \\
(\mathrm{n}=74, \text { unless otherwise stated })\end{array}$} & \multicolumn{2}{|l|}{$\begin{array}{l}\text { Multivariable analysis } \\
\mathrm{R}^{2}=0.139, \mathrm{n}=71\end{array}$} \\
\hline & $\begin{array}{l}\text { Correlation } \\
\text { coefficient }\end{array}$ & B $(95 \% \mathrm{CI})$ & $\mathrm{p}$ value & $\mathrm{B}(95 \% \mathrm{CI})$ & $\mathrm{p}$ value \\
\hline Age* & 0.152 & $0.075(-0.039,0.190)$ & 0.195 & $0.079(-0.041,0.200)$ & 0.191 \\
\hline Male sex* & -0.281 & $-2.897(-5.219,-0.574)$ & 0.015 & $-3.246(-5.629,-0.863)$ & 0.008 \\
\hline Systolic blood pressure* & 0.107 & $0.025(-0.030,0.080)$ & 0.365 & $0.022(-0.036,0.081)$ & 0.452 \\
\hline Ever smoked* & 0.034 & $0.314(-1.847,2.474)$ & 0.773 & $0.069(-2.048,2.185)$ & 0.948 \\
\hline Body mass index & 0.092 & $0.129(-0.200,0.458)$ & 0.437 & - & - \\
\hline Waist/hip circumference & -0.022 & $-1.279(15.390,12.833)(\mathrm{n}=72)$ & 0.857 & - & - \\
\hline TC/HDL-C* & 0.072 & $0.319(-0.746,1.384)(\mathrm{n}=71)$ & 0.553 & $0.333(-0.698,1.363)$ & 0.521 \\
\hline HOMA-IR & -0.008 & $-0.017(-0.522,0.489(n=69) \dagger$ & 0.410 & - & - \\
\hline NT-proBNP & 0.064 & $0.004(-0.012,0.020)(\mathrm{n}=69)$ & 0.850 & - & - \\
\hline RA disease duration & -0.024 & $-0.011(-0.115,0.094)$ & 0.851 & - & - \\
\hline 3 Variable DAS28 & 0.047 & $0.169(-0.674,1.012)$ & 0.690 & - & - \\
\hline ACPA & 0.020 & $0.235(-2.523,2.994)(\mathrm{n}=73)$ & 0.866 & - & - \\
\hline HAQ-DI & -0.050 & $-0.293(-1.722,1.136)(\mathrm{n}=70)$ & 0.448 & - & - \\
\hline History of joint surgery & -0.001 & $-0.014(-2.398,2.369)$ & 0.990 & - & - \\
\hline Current use of biological-DMARD & 0.128 & $1.250(-1.027,3.527)$ & 0.277 & - & - \\
\hline
\end{tabular}

Bold values represent statistical significance in multivariable analysis

$A C P A$ anti-citrullinated peptide antibody, $C R P$ C-reactive protein, DAS28CRP 28 joint disease activity score, DMARD disease-modifying antirheumatic drug, HAQ-DI health assessment questionnaire-disability index, HOMA-IR homeostasis model of assessment of insulin resistance, $L V E F$ left ventricular ejection fraction, $N T$-proBNP N-terminal pro-brain natriuretic peptide, $S$ ' peak systolic strain rate, $T C / H D L-C$ total cholesterol/high-density lipoprotein cholesterol ratio

*Entered into linear regression model as associated with LVEF in the literature

$\dagger$ Excluding high outlier: Correlation coefficient 0.108 B $0.521(95 \% \mathrm{CI}-0.585,1.627) \mathrm{p}=0.350$

with the treatment of RA in the biologic era. Further work is required to understand the underlying pathophysiology, particularly of the reduced LVMI, including any relationship with RA-cachexia. There is also a need for the development of effective cardiovascular risk reduction programs. We also acknowledge defining subclinical cardiovascular disease using CMR imaging is currently difficult given the multiple domains/measurements. A future area of focus for crossspeciality collaborations such as ours should be to define how subclinical disease is reported.

\section{Conclusions}

In summary, this CMR study has demonstrated that patients with established RA and no history of CVD have evidence of reduced LV systolic function and LVMI after adjustment for traditional cardiovascular risk factors. The reduction in LVMI suggests cardiac pathology other than atherosclerosis in RA. Traditional cardiovascular risk factors appear to be key determinants of subclinical CVD in RA potentially warranting more effective cardiovascular risk reduction programs.

Acknowledgements We would like to acknowledge the LIRRM and LICAMM research nursing and administrative staff within the University of Leeds, particularly Petra Bijsterveld (senior cardiology research nurse) and Oliver Wordsworth (rheumatology research nurse), who helped conduct this study. Support was received from the Leeds NIHR Clinical Research Facility. The views expressed are those of the author(s) and not necessarily those of the NHS, the NIHR or the Department of Health.

Funding This study was funded internally and supported by the National Institute for Health Research (NIHR) Leeds Biomedical Research Centre and NIHR Leeds Clinical Research Facility. The views expressed are those of the authors and not necessarily those of the NHS, NIHR or the Department of Health.

\section{Compliance with ethical standards}

Conflict of interest The authors declare no conflicts in interest.

Ethical approval The work within this study was approved by the Leeds West ethics committee (REC 09/H1307/98 and REC 10/H1307/103) and have therefore been performed in accordance with the ethical 
standards laid down in the 1964 Declaration of Helsinki and its later amendments.

Informed consent All persons gave their informed consent prior to their inclusion in the study.

Open Access This article is licensed under a Creative Commons Attribution 4.0 International License, which permits use, sharing, adaptation, distribution and reproduction in any medium or format, as long as you give appropriate credit to the original author(s) and the source, provide a link to the Creative Commons licence, and indicate if changes were made. The images or other third party material in this article are included in the article's Creative Commons licence, unless indicated otherwise in a credit line to the material. If material is not included in the article's Creative Commons licence and your intended use is not permitted by statutory regulation or exceeds the permitted use, you will need to obtain permission directly from the copyright holder. To view a copy of this licence, visit http://creativecommons.org/licenses/by/4.0/.

\section{References}

1. Innala L, Moller B, Ljung L, Magnusson S, Smedby T et al (2011) Cardiovascular events in early RA are a result of inflammatory burden and traditional risk factors: a 5 year prospective study. Arthritis Res Ther 13(4):R131

2. Agca R, Heslinga SC (2017) EULAR recommendations for cardiovascular disease risk management in patients with rheumatoid arthritis and other forms of inflammatory joint disorders: 2015/2016 update. Ann Rheum Dis 76(1):17-28. https://doi. org/10.1136/annrheumdis-2016-209775

3. Greenwood JP, Maredia N, Younger JF, Brown JM, Nixon J et al (2012) Cardiovascular magnetic resonance and single-photon emission computed tomography for diagnosis of coronary heart disease (CE-MARC): a prospective trial. Lancet 379(9814):453460. https://doi.org/10.1016/S0140-6736(11)61335-4

4. Kobayashi Y, Giles JT, Hirano M, Yokoe I, Nakajima Y et al (2010) Assessment of myocardial abnormalities in rheumatoid arthritis using a comprehensive cardiac magnetic resonance approach: a pilot study. Arthritis Res Ther 12(5):R171

5. Kobayashi Y, Isamu Y, Hirano M, Kobayashi H (2012) Detection of left ventricular regional dysfunction using strain analysis by feature tracking of Cine MRI in rheumatoid arthritis patients without cardiac symptoms. J Cardiovasc Magn Reson 14(1):P191

6. Mavrogeni S, Sfikakis PP, Gialafos E, Bratis K, Karabela G et al (2014) Cardiac tissue characterization and the diagnostic value of cardiovascular magnetic resonance in systemic connective tissue diseases. Arthritis Care Res 66(1):104-112. https://doi. org/10.1002/acr.22181

7. Mavrogeni S, Karabela G, Stavropoulos E, Gialafos E, Sfendouraki E et al (2013) Imaging patterns of heart failure in rheumatoid arthritis evaluated by cardiovascular magnetic resonance. Int J Cardiol 168(4):4333-4335. https://doi.org/10.1016/j.ijcar d.2013.05.085

8. Ntusi NA, Piechnik SK, Francis JM, Ferreira VM, Matthews PM et al (2015) Diffuse myocardial fibrosis and inflammation in rheumatoid arthritis: insights from CMR T1 mapping. JACC Cardiovasc Imaging 8(5):526-536. https://doi.org/10.1016/j. jcmg.2014.12.025

9. Giles JT, Malayeri AA, Fernandes V, Post W, Blumenthal RS et al (2010) Left ventricular structure and function in patients with rheumatoid arthritis, as assessed by cardiac magnetic resonance imaging. Arthritis Rheum 62(4):940-951
10. Erhayiem B, McDiarmid A, Swoboda P, Kidambi A, Ripley D et al (2015) OP0163 treatment-naïve, early rheumatoid arthritis Patients demonstrate vascular and myocardial abnormalities on cardiac MRI. Ann Rheum Dis 74(Suppl 2):130-131. https://doi. org/10.1136/annrheumdis-2015-eular.5279

11. Aslam F, Bandeali SJ, Khan NA, Alam M (2013) Diastolic dysfunction in rheumatoid arthritis: a meta-analysis and systematic review. Arthritis Care Res 65(4):534-543. https://doi.org/10.1002/ acr.21861

12. Kobayashi H, Kobayashi Y, Giles JT, Yoneyama K, Nakajima Y et al (2014) Tocilizumab treatment increases left ventricular ejection fraction and decreases left ventricular mass index in patients with rheumatoid arthritis without cardiac symptoms: assessed using 3.0 tesla cardiac magnetic resonance imaging. J Rheumatol 41(10):1916-1921. https://doi.org/10.3899/jrheum.131540

13. Crowson CS, Nicola PJ, Kremers HM, O'Fallon WM, Therneau TM et al (2005) How much of the increased incidence of heart failure in rheumatoid arthritis is attributable to traditional cardiovascular risk factors and ischemic heart disease? Arthritis Rheum 52(10):3039-3044. https://doi.org/10.1002/art.21349

14. Arnett FC, Edworthy SM, Bloch DA, McShane DJ, Fries JF et al (1988) The American Rheumatism Association 1987 revised criteria for the classification of rheumatoid arthritis. Arthritis Rheum 31(3):315-324

15. Smolen JS, Breedveld FC, Eberl G, Jones I, Leeming M et al (1995) Validity and reliability of the twenty-eight-joint count for the assessment of rheumatoid arthritis activity. Arthritis Rheum 38(1):38-43

16. Fries JF, Spitz P, Kraines RG, Holman HR (1980) Measurement of patient outcome in arthritis. Arthritis Rheum 23(2):137-145

17. Matthews DR, Hosker JP, Rudenski AS, Naylor BA, Treacher DF et al (1985) Homeostasis model assessment: insulin resistance and beta-cell function from fasting plasma glucose and insulin concentrations in man. Diabetologia 28(7):412-419

18. Alfakih K, Plein S, Thiele H, Jones T, Ridgway JP et al (2003) Normal human left and right ventricular dimensions for MRI as assessed by turbo gradient echo and steady-state free precession imaging sequences. J Magn Reson Imaging 17(3):323-329. https ://doi.org/10.1002/jmri.10262

19. Burns J, Sivananthan MU, Ball SG, Mackintosh AF, Mary DASG et al (2007) Relationship between central sympathetic drive and magnetic resonance imaging-determined left ventricular mass in essential hypertension-response. Circulation 116(17):E417E417. https://doi.org/10.1161/Circulationaha.107.725903

20. Lee JM, Shirodaria C, Jackson CE, Robson MD, Antoniades C et al (2007) Multi-modal magnetic resonance imaging quantifies atherosclerosis and vascular dysfunction in patients with type 2 diabetes mellitus. Diab Vasc Dis Res 4(1):44-48. https://doi. org/10.3132/dvdr.2007.005

21. Messroghli DR, Bainbridge GJ, Alfakih K, Jones TR, Plein S et al (2005) Assessment of regional left ventricular function: accuracy and reproducibility of positioning standard short-axis sections in cardiac MR imaging. Radiology 235(1):229-236. https://doi. org/10.1148/radiol.2351040249

22. Messroghli DR, Greiser A, Frohlich M, Dietz R, Schulz-Menger J (2007) Optimization and validation of a fully-integrated pulse sequence for modified look-locker inversion-recovery (MOLLI) T1 mapping of the heart. J Magn Reson Imaging 26(4):10811086. https://doi.org/10.1002/jmri.21119

23. Messroghli DR, Radjenovic A, Kozerke S, Higgins DM, Sivananthan MU et al (2004) Modified Look-Locker inversion recovery (MOLLI) for high-resolution T-1 mapping of the heart. Magnet Reson Med 52(1):141-146. https://doi.org/10.1002/mm.20110

24. Schulz-Menger J, Bluemke DA, Bremerich J, Flamm SD, Fogel MA et al (2013) Standardized image interpretation and post processing in cardiovascular magnetic resonance: society for 
cardiovascular magnetic resonance (SCMR) board of trustees task force on standardized post processing. J Cardiovasc Magn Reson 15(1):35

25. Oliver JJ, Webb DJ (2003) Noninvasive assessment of arterial stiffness and risk of atherosclerotic events. Arterioscl Throm Vas 23(4):554-566

26. Ibrahim ESH, Johnson KR, Miller AB, Shaffer JM, White RD (2010) Measuring aortic pulse wave velocity using high-field cardiovascular magnetic resonance: comparison of techniques. J Cardiovasc Magn Reson 12(1):26

27. Ripley DP, Negrou K, Oliver JJ, Worthy G, Struthers AD et al (2015) Aortic remodelling following the treatment and regression of hypertensive left ventricular hypertrophy: a cardiovascular magnetic resonance study. Clin Exp Hypertens 37(4):308-316. https://doi.org/10.3109/10641963.2014.960974

28. Swoboda PP, Larghat A, Zaman A, Fairbairn TA, Motwani M et al (2014) Reproducibility of myocardial strain and left ventricular twist measured using complementary spatial modulation of magnetization. J Magn Reson Imaging 39(4):887-894. https:// doi.org/10.1002/jmri.24223

29. Musa TA, Uddin A, Swoboda PP, Fairbairn TA, Dobson LE et al (2017) Cardiovascular magnetic resonance evaluation of symptomatic severe aortic stenosis: association of circumferential myocardial strain and mortality. J Cardiovasc Magn Reson 19(1):13. https://doi.org/10.1186/s12968-017-0329-7

30. McDiarmid AK, Broadbent DA, Higgins DM, Swoboda PP, Kidambi A et al (2015) The effect of changes to MOLLI scheme on T1 mapping and extra cellular volume calculation in healthy volunteers with 3 tesla cardiovascular magnetic resonance imaging. Quant Imaging Med Surg 5(4):503-510. https://doi.org/10.3978/j. issn.2223-4292.2015.04.07

31. Holm S (1979) A simple sequentially rejective multiple test procedure. Scand J Stat 6(2):65-70

32. Corrao S, Argano C, Pistone G, Messina S, Calvo L et al (2015) Rheumatoid arthritis affects left ventricular mass: systematic review and meta-analysis. Eur J Intern Med 26(4):259-267. https ://doi.org/10.1016/j.ejim.2015.02.008

33. Midtbo H, Semb AG, Matre K, Kvien TK, Gerdts E (2017) Disease activity is associated with reduced left ventricular systolic myocardial function in patients with rheumatoid arthritis. Ann Rheum Dis 76(2):371-376. https://doi.org/10.1136/annrheumdi s-2016-209223

34. Bellenger NG, Burgess MI, Ray SG, Lahiri A, Coats AJ et al (2000) Comparison of left ventricular ejection fraction and volumes in heart failure by echocardiography, radionuclide ventriculography and cardiovascular magnetic resonance; are they interchangeable? Eur Heart J 21(16):1387-1396. https://doi. org/10.1053/euhj.2000.2011
35. Bellenger NG, Davies LC, Francis JM, Coats AJ, Pennell DJ (2000) Reduction in sample size for studies of remodeling in heart failure by the use of cardiovascular magnetic resonance. J Cardiovasc Magn Reson 2(4):271-278

36. Armstrong AC, Gidding S, Gjesdal O, Wu C, Bluemke DA et al (2012) LV mass assessed by echocardiography and CMR, cardiovascular outcomes, and medical practice. JACC Cardiovasc Imaging 5(8):837-848. https://doi.org/10.1016/j.jcmg.2012.06.003

37. Ciftci O, Yilmaz S, Topcu S, Caliskan M, Gullu H et al (2008) Impaired coronary microvascular function and increased intima-media thickness in rheumatoid arthritis. Atherosclerosis 198(2):332-337. https://doi.org/10.1016/j.atherosclerosis .2007.11.013

38. Cohn JN, Ferrari R, Sharpe N (2000) Cardiac remodeling-concepts and clinical implications: a consensus paper from an international forum on cardiac remodeling. Behalf of an International Forum on Cardiac Remodeling. J Am Coll Cardiol 35(3):569-582

39. Myasoedova E, Davis JM 3rd, Crowson CS, Roger VL, Karon BL et al (2013) Brief report: rheumatoid arthritis is associated with left ventricular concentric remodeling: results of a populationbased cross-sectional study. Arthritis Rheum 65(7):1713-1718. https://doi.org/10.1002/art.37949

40. Summers GD, Deighton CM, Rennie MJ, Booth AH (2008) Rheumatoid cachexia: a clinical perspective. Rheumatology 47(8):1124-1131. https://doi.org/10.1093/rheumatology/ken146

41. Piepoli MF, Hoes AW, Agewall S, Albus C, Brotons C et al (2016) 2016 European Guidelines on cardiovascular disease prevention in clinical practice: the Sixth Joint Task Force of the European Society of Cardiology and Other Societies on Cardiovascular Disease Prevention in Clinical Practice (constituted by representatives of 10 societies and by invited experts) developed with the special contribution of the European Association for Cardiovascular Prevention \& Rehabilitation (EACPR). Eur Heart J 37(29):23152381. https://doi.org/10.1093/eurheartj/ehw106

42. Kitas GD, Nightingale P, Armitage J, Sattar N, Belch JJF et al (2015) Trial of atorvastatin for the primary prevention of cardiovascular events in patients with RA (TRACE RA): a randomized trial in 2986 RA patients (094). Rheumatology 54(suppl 1):i87. https://doi.org/10.1093/rheumatology/kev088.094

43. Scott IC, Ibrahim F, Johnson D, Scott DL, Kingsley GH (2012) Current limitations in the management of cardiovascular risk in rheumatoid arthritis. Clin Exp Rheumatol 30(2):228-232

Publisher's Note Springer Nature remains neutral with regard to jurisdictional claims in published maps and institutional affiliations. 